Interactive comment on "Implementation of aerosol-cloud interactions in the regional atmosphere-aerosol model COSMO-MUSCAT and evaluation using satellite data" by Dipu Sudhakar et al.

Dipu Sudhakar et al.

dipu.sudhakar@uni-leipzig.de

Received and published: 15 December 2016

\title{
Reviewer Comments:2
}

Major Revisions:

-Whilst the paper gives a detailed introduction of the general benefits of regional models with online coupled aerosol-cloud, aerosol-radiation and aerosol-cloudradiation interactions, such as WRF-CHEM (Grell et al. 2005), COSMO-ART (Bangert 
et al. 2011), COSMO-M7 (Zubler et al. 2011) and COSMO-ART/M7 (chapter 2 of: http://e-collection.library.ethz.ch/eserv/eth:48845/eth-48845-02.pdf), I would like to be given further motivation regarding the advantages of their particular approach and have it contrasted to existing approaches.

My questions raised here, could guide such a discussion:

1) Why did you use the Boucher and Lohmann's (1995) empirical relation, rather than implementing newer approaches using Koehler theory that have previously been applied in COSMO-ART/COSMO-M7 such as Abdul-Razzak \& Gan (2000), or Nenes \& Seinfeld (2003)? What is your justification for only considering sulfate and ignoring nitrate contributions to CCN?

ANS: Our objective is to replace constant $C_{c c n}$ in COSMO model with $C_{c c n}$ from MUSCAT model. This particular version of COSMO-MUSCAT (Version 5.0) is only available with aerosol mass concentration for different aerosol species. As an initial stage of two-moment scheme modification, we have used Boucher and Lohmann parameterization to convert sulfate aerosol mass concentration to $C_{c c n}$ number concentration. In the next step, M7 will be implemented and we could directly use aerosol number concentration in the two-moment scheme, along with new approaches.

2) Whilst the relationship between cloud optical depth, effective radius and vertically integrated liquid water content is a commonly used diagnostic, it is wavelength independent. How did you deal with this issue when implementing this approach in a radiation scheme that computes the radiative transfer within 3 SW and 5 LW bands covering the entire wavelength spectrum?

Printer-friendly version

ANS: Even though the cloud optical properties are wavelength independent, the extinction coefficients are calculated from wavelength dependents variables. The

Discussion paper

C2 
extinction, scattering and asymmetry parameters are depends on entire wavelength spectra (Zubler et al., (2011)).

- Furthermore, a short motivation should be included regarding the chosen case study. Are all the clouds shown boundary layer stratocumulus?

ANS: A short motivation is included in the manuscript. All clouds are not boundary layer stratocumulus, it consists of convective clouds too.

- The model evaluation is performed on a single day. The authors argue that the forecast skill decreases with increasing lead time. Could you obtain better agreement and therefore obtain a longer evaluation period if you performed nudged simulations? Otherwise, a brief justification should be given that 1 day is a sufficiently long time period for your evaluation.

ANS: Even though the model comparison via spatial distribution is performed only for a single day, more quantitative analysis (PDF comparison for single day and for entire simulation) has been carried out and included in the manuscript (Figure 5). The nudged simulation may improve the evaluation, however in this first stage we are mainly focusing on COSMO-MUSCAT coupling and COSP satellite simulator incorporation and its comparison with observations. A brief justification is included in the manuscript.

\section{General Comments:}

Printer-friendly version

Discussion paper

- This paper talks about the coupling of aerosol-cloud interactions and aerosol-cloudradiation interactions. However, it should be stated clearly (already in the abstract) 
that these interactions are only included explicitly for warm-phase cloud processes.

ANS: These interactions are applied for liquid water clouds due to complexity of the aerosol cloud interaction in ice clouds, which is mentioned in the manuscript.

- The paper often talks about changes to the COSMO model in general. However, the modifications are applied not to the COSMO model in general, but to the COSMO version of COSMO-MUSCAT. Whilst I agree with the authors that CCN variability and aerosol-cloud-radiation coupling is not provided in the officially released code versions, such developments have already been included in other COSMO versions (COSMO-ART, COSMO-M7, COSMO-ART/M7). They are therefore not new to the COSMO code itself.

ANS: Although, different COSMO versions (COSMO-ART, COSMO-M7, COSMOART/M7) have been modified, these models used different cloud microphysics (mainly droplet activation), for example in COSMO-ART the droplet activation based on Bangert et al., (2001), which replaces the activation rate of equation (17) of Seifert et al., (2006) and in COSMO-M7, it is treated with following parameterizations Leaitch et al. (1996), Lin and Leaitch (1997), and Lohmann (2002).

- The paper is written in good scientific English. However, some sentences require re-writing (some of which are listed below). Furthermore, articles are missing in a few places.

\section{Specific Comments:}

- P2L6: Last sentence needs rewriting.

ANS: Revised as suggested 
- P2L15: Sentence needs rewriting:"This approach, however,...”

ANS: Revised as suggested

- P2L31-32: Delete last sentence of this paragraph. It is mis-leading as all of the mentioned aerosol-coupled regional models include a coupling of the cloud microphysics to the radiation.

ANS: Revised as suggested

- P3L22:Sentence needs rewriting:"In this study, the COSMO model..." Equation 5: Change $\frac{1.5}{2}$ to $\frac{3}{4}$.

ANS: In this study, COSMO model has been configured in a non convection permitting mode with uniform horizontal grid resolution of $0.25^{\circ}(\approx 28 \mathrm{~km})$.

$$
\tau_{c}=\frac{3 \rho q_{c} d z}{2 \rho_{w} r_{e}}
$$

- P4L23: Sentence needs rewriting: "IN the COSMO model, the aerosol..."

ANS: Revised as suggested

- P5L1-11: This discussion needs some clarification: How is the Smax issue raised overcome? You raise the issue of ignoring updraft velocity in the Boucher and Lohmann formulation, however, is this then not introduced by $d S / d z^{*} w$ in Eq.7 (which admittedly is a very simplistic formulation of this relationship).

ANS: In this case simulations are carried out for intermediate aerosol condition, in which $S_{\max }$ is set at $2.0 \%$. Boucher and Lohmann parameterization is bound to updraft velocity uncertainty, however it can be overcome by $d S / d z^{*} w$ in Eq.7. This part is revised.

- P5L20ff: Here you list all satellite products used for evaluation. Also include CERES

Printer-friendly version

Discussion paper 
here, as you use it later.

ANS: Revised as suggested

- P5L27: I suggest to delete: In the upcoming... You have 1 ISCCP figure, 2 Modis and 1 CERES. That is not massively unbalanced.

ANS: Revised as suggested

- P5L30: I suggest to delete last sentence of this paragraph.

ANS: Revised as suggested

- P5L25-30: Please rephrase. The point regarding satellite biases could be formulated like: "One should keep in mind that the satellite products, just like models, are prone to biases. Comparisons of satellite retrievals with ... have shown that... Nonetheless, spatial correlation of the cloud structures are well represented."

ANS: Revised as suggested

- P6L2: Sentence needs rewriting: "As the forecast time..."

ANS: Revised as suggested

- P6L10: Results are only shown for 17th. Not 15th-25th. Please include description of the cloud types of this domain (altitude, phase, average thickness, surface precipitation). When did you start your simulation, cause the 2nd day would be the 16 th if the simulation were started in the morning of the 15th? Also, why are is the 16th not included in the analysis?

ANS: In the revised manuscript, synoptic conditions for 15 Feb (Figure S1) and 17 Feb (Figure 2) are included along with cloud type. Simulation started at $15 \mathrm{Feb}, 00.00 \mathrm{hrs}$, then 17-Feb would be the third day of the simulation (Revised in the manuscript).

Printer-friendly version

Discussion paper 
- P6L22-25: First 3 sentences should go into section 2.1.1 (methods).

ANS: Revised as suggested

- P7L1-4: First 3 sentences should go into section 2.1.1 (methods).

ANS: Revised as suggested

- P7L6: "The top panel shows...." This should be in Fig. Caption.

ANS: Revised as suggested

- P7L 19: Please check units.

ANS: The unit has been corrected to $\mathrm{kgm}^{-2}$

- P7L25-30: In this discussion quantitative statements should be included. For instance, the area mean changes + variability could be determined. It would help determine the signal from the noise in figures $g$ to $i$.

ANS: For quantitative comparison, PDF distribution of cloud products are included in the analysis.

- P8L 14: First sentence of paragraph need rewriting/clarification.

ANS: Sentence rephrased: While comparing modified two-moment scheme results with MODIS level-2 satellite products, the model shows more cloud free (clear) grid points. This indicates that model is unable to capture the sub grid scale cloud patterns accurately (Jason and Thomas, 2008), which may be due to the coarse resolution $\left(0.25^{\circ}\right)$ of the model.

Printer-friendly version

- P8L19: Maybe rephrase title, because aerosol-cloud radiation interactions were already discussed in previous section (cloud optical thickness). Suggestion: "Impacts

Discussion paper 
on radiative balance" In general this discussion is not very precise. It would help the discussion if you relate observed changes in e.g. the SW fluxes to the decrease in cloud optical thickness...

ANS: Revised as suggested

- P8L30: Please rephrase. It is an initial approach to modify COSMO-MUSCAT only. ANS: Revised as suggested

- P9L7: Finding 1 should be removed as it is not discussed in the paper.

ANS: Finding 1 has been changed to: "The modified two-moment scheme results have been compared with two-moment version of COSMO model. In terms of the cloud distributions, this modification has only a minor effect".

- P9L13: Paragraph missing after point 2.

ANS: Revised as suggested

- P9L22: Last sentence needs rewriting and one reference is missing.

ANS: Sentence rephrased: This can result in more precise cloud droplet activation parameterization, involving different aerosol species as $\mathrm{CCN}$, and thus improving the cloud droplet number calculation of Lohmann et al., (2007).

- P10ff: A few references need changing. Some paper titles use capital letters. IPCC reference is incomplete

ANS: Revised as suggested

Printer-friendly version

- Fig1: More detail should be given in the caption. I think this figure could be

Discussion paper 
improved/clarified. Why are the "emissions/land use" and M7 stand-alone and not connected? The figure could be clearer regarding the structure of the code. Are RACM and MUSCAT separate modules? Will M7 not be embedded in MUSCAT? Is the output really completely separate?

ANS: Figure 1 has been modified, COSMO-MUSCAT is an online coupled modeling system, which uses COSMO meteorological fields to drive the model. Emissions and land use data are in input for MUSCAT model and RACM is a module, which is included in MUSCAT. In this particular version of COSMO-MUSCAT(V5.0), M7 not yet embedded. Also, the outputs are separate.

- Fig2: I personally would not include all "H" and "L" markes. I would simply mark the center of the dominant low pressure and high pressure systems.

ANS: Revised as suggested

- Fig3/Fig4: Looking at these figures optically, I would arrive at different cloud fractions for Fig3b and Fig4d. Areas where the total cloud fraction of the model is $100 \%$, the diagnosed optical thickness is 0 or just very small? Does the COSP simulator include subgrid-scale cloud water? If not, is that justified for cloud optical thickness?

ANS: ISCCP satellite observations are available with $280 \mathrm{~km}$ resolution, for the comparison COSP derived ISCCP output $(28 \mathrm{~km})$ are re-gridded to $280 \mathrm{~km}$, which is stated in the manuscript. The cloud water simulated at sub-column within model grids account for subgridâĂšscale variability.

- Fig4: Maybe regional/domain means + a measure of variability could be given to highlight the results more quantitatively?

ANS: For quantitative comparison, PDFs are used, instead of regional means, which may add uncertainty.

Printer-friendly version

Discussion paper 
- Fig5: What is going on with the MODIS cloud droplet number estimate? Why is the spatial pattern so different than in Fig4a-c. Are these clouds just too shallow to obtain a good number estimate?

ANS: CDNC can be derived from MODIS cloud optical depth $\tau_{c}$ and effective radius $r_{e}$ (Quaas et al., 2009), which is given by,

$$
N_{d}=\alpha \tau_{c}^{0.5} r_{e}^{-2.5}
$$

where $\alpha=1.37 \times 10^{-5} m^{-0.5}$. In the above equation the lower limit of $\tau_{c}$ and $r_{e}$ are constrained to 5 and 2 . This would result in low CDNC in figure $6 \mathrm{c}$.

- Fig6/Fig7: I find the color scale depicting the fluxes very misleading. I would suggest using a pure blue color scale for $L W$ fluxes and pure red for SW fluxes.

ANS: Revised as suggested

Interactive comment on Geosci. Model Dev. Discuss., doi:10.5194/gmd-2016-186, 2016. 